\title{
LA CRISIS EN IRLANDA DEL NORTE
}

BRIAN PETER HUGHES

Universidad de Alicante

Cuando uno considera el estado actual de la sociedad norirlandesa, la primera impresión es de perplejidad: la violencia sectaria, el fanatismo religioso, la intolerancia y la manipulación políticas, una pobreza acentuada y aparentemente incurable, y una estructura económica más de acuerdo con modelos tercermundistas que con los de Europa Occidental son algunos de los rasgos más acusados. Esto, unido a las maniobras imprevisibles, y a veces manifiestamente turbias, de Gran Bretaña y Eire, produce en el observador no iniciado una serie de reacciones que van desde la inquietud o lástima, hasta la incredulidad, el desconcierto y la indignación. Por ello, puede que sirva de consuelo consignar desde el principio que la autopercepción de algunos irlandeses les hace reconocer, como característica heredada, el elemento de terquedad y contradicción temperamentales, de obstinación extremada y malintencionada, evidentes en el comportamiento de algunos norirlandeses desde hace unos quince años.

Por ejemplo, el conocido crítico y ensayista Denis Donoghue ha escrito lo siguiente, refiriéndose a sus compatriotas:

Entre otras razones, el pensamiento político resulta especialmente difícil en Irlanda no tanto porque existe odio entre unos cuantos católicos y unos cuantos protestantes, sino porque aquí el pensamiento está ahogado por el mito, por la sensación de que ahora es tarde para pensar - la naturaleza ya lo ha pensado todo'

1 The New Statesman, 22 de septiembre, 1978, pág. 366. 
Una observación similar, aunque tendenciosa por lo cínica, la ha hecho el historiador A. T. Q. Stewart:

Para los irlandeses, la Historia entera es Historia Aplicada: el pasado no es más que una cantera que les viene muy bien, porque de ella sacan argumentos que utilizan contra sus enemigos en el presente. En la Historia como tal tienen muy poco interés. Entonces, al decir que los irlandeses están demasiado influenciados por el pasado, lo que queremos decir en realidad es que están demasiado influenciados por la historia irlandesa, que es algo bien distinto ${ }^{2}$.

Para profundizar más en la psicología del habitante de Irlanda de Norte, citemos a dos poetas nativos, el primero católico y protestante el segundo. Seamus Heaney ha descrito «la famosa/ /Reticencia norteña, la apretada mordaza de lugar/Y tiempo. Sí, sí. Canto a los seis condados del país nuestro/En donde, para salvarte, lo único que debes salvar son las apariencias; $/ Y$, digas lo que digas, no digas nada ${ }^{3}$.

A estos versos les da una réplica la caracterización de la "Provincia» y sus habitantes en el estilo seco y guasón de W. R. Rodgers: "Un pueblo hosco, áspero y protestante/Para quien la palabra es siempre palabra de combate,/Cuya ira se muestra en silencio/Ante la labia fácil y oronda de los sureños./Los míos no tuvieron tanta suerte al nacer, ni tal pico de oro/Ni te habrían de agradecer que les dieses el don de lenguas;/La respuesta irónica, la réplica amarga/Son el pan nuestro del norteño/Y su largo trago es el silencion'.

Naturalmente, toda generalización es peligrosa, y probablemente sea inútil, a la larga, intentar captar y definir los rasgos característicos de un pueblo. Sin embargo, estas dos citas sí parecen poseer un sentido general coherente, identificado como rasgos de la "psique" norirlandesa, por un lado cierta pasión por el rito y cierta obsesión por el pasado tribal, y por otro, un sentido del humor cerrado, retorcido, socarrón. La cuestión tiene su

2 The Narrow Ground, Londres, Faber, 1977, pág. 16.

3 Seamus Heaney, "Whatever You Say, Say Nothing" en North, Londres, Faber, 1975.

${ }^{4}$ W. R. Rodgers, "The Character of Ireland", en Collected Poems, Oxford, 1967. 
importancia tanto por la luz que arroja sobre las actitudes de este pueblo, como por el hecho de que la idea misma de su identidad racial o cultural es de sumo interés a la hora de juzgar los actos y las aspiraciones de los habitantes actuales del nordeste de Irlanda. Quiérese decir que una comprensión cabal de la crisis de dicha región y, venido el caso, cualquier posibilidad dè llegar a una solución duradera, dependen de una percepción clara y precisa de quiénes son estas gentes, y qué es lo que las divide.

Dentro de los Seis Condados ${ }^{5}$, se ha dado tres respuestas distintas a esta pregunta. Los dos pueblos (o dos naciones, como a veces se dice) son "Plantador" y Gael (es decir, colonos y nativos) o bien los del "Naranja" y los del "Verde" (entiéndase: protestantes y católicos) o, por último opresores y oprimidos, según sea la explicación ofrecida de tipo histórico, religioso o político, y con arreglo al punto de vista personal de quien la ofrezca.

Históricamente, el primero de estos binomios se remonta a los primeros intentos sistemáticos por parte de Inglaterra de subyugar y "apaciguar" (es decir: anexionar) a Irlanda, política iniciada por Isabel I en el siglo XVI y concluida en el XVII por Cromwell. (En algunas zonas del pais, hoy todavia, este apellido no se distingue claramente de los múltiples apelativos del Demonio, y es frecuente que su pronunciación vaya seguida de un sonoro escupitajo.) El presbiterianismo virulento de los colonos de dicho periodo, escoceses en su mayoría, continúa siendo reflejado en términos de la sociología contemporánea, ya que las

5 En el presente trabajo, y en la medida de lo posible, se evita por inexacto y ahistórico, el uso del término U/ster para referirnos a la entidad politica de la que se trata. Ulster, junto a Leinster, Munster y Connaught, era uno de los cuatro reinos de la Irlanda antigua y, como ellos, pasó más tarde a ser provincia administrativa. Constaba de los seis condados actuales (Antrim, Down, Derry, Fermanagh, Armagh y Tyrone) además de Monaghan, Cavan y Donegal. A raíz de la partición de Irlanda en 1920, una redefinición del Ulster, que hay que calificar de fraudulenta, excluyó a los tres últimos, porque la mayoría católica de sus poblaciones habría amenazado a la mayoria pro-unionista permanente que pretendian asegurar los sagaces autores de la división. Asimismo, el prefijar "London" al nombre de la ciudad y condado de Derry es de origen colonial, carece de justificación histórica y nunca ha encontrado aceptación por parte de los irlandeses indigenas. Por tanto, no lo emplearemos tampoco. 
zonas de población con mayoría protestante más amplia, y con acumulaciones más considerables de riqueza y privilegio, se encuentran todavía en los condados de Antrim y Down, que son los que están más al este, frente a Escocia.

Estos hechos, a su vez, contribuyen a explicar la hostilidad mutua que enfrenta a católicos (descendientes de los Gaeles primitivos) y protestantes (descendidos, en gran parte, de los colonos isabelinos), enfrentamiento que se ha consolidado en la división entre quienes apoyan al Verde de la bandera nacionalista tradicional, y los que se unen al Naranja ${ }^{6}$. En cuanto al tercer binomio, opresores/oprimidos, es claro que pretende sustituir a la percepción religiosa por otra socio-política, condenando de manera explícita la conquista de la Isla de Irlanda, a la que tacha de invasión colonial oportunista con fines exclusivamente económicos. En la actualidad, empero, la opción socio-política, más que superar a la religiosa, parece haberla subsumido.

Sintetizando, parece bastante evidente que, aunque el primero y el tercero de estos binomios concuerdan en tanto en cuanto ambos ven en Irlanda del Norte el resultado de una política expansionista, en el fondo representan actitudes distintas: aquél es un término puramente descriptivo, mientras que éste, por bien planteado que esté, contiene un elemento emotivo, e incluso injurioso. En el momento actual, la distinción entre Colono y Gael la asumirían tanto los nacionalistas como los unionistas, mientras que la que opone opresores a oprimidos es indefectiblemente la prerrogativa del anti-unionista a ultranza. En cuanto al segundo binomio, que distingue entre católicos y protestantes - o, en lengua vernácula, entre "Papists" y "Tagues" 0 "Fenians", por una parte, y "Prods", "Billies" o "Orangemen", por otra - quizá habría que ver en él una visión popular, vestigial pero duradera, de los resultados de la política isabelina, una especie de guía, improvisada y expeditiva pero eficaz, que permite identificar a las partes en conflicto en Irlanda del Norte.

6 En el levantamiento de la semana de Pascua de 1916, los insurrectos portaban una bandera tricolor: verde (simbolo nacionalista), naranja (en representación de la minoria protestante del Norte) y blanca (emblema de la deseada unión entre los pueblos). Dicha bandera es hoy día la enseña nacional de Eire. 
Como el propósito del presente trabajo es examinar la crisis en la sociedad norirlandesa contemporánea, y sus efectos sobre el contexto hiberno-británica, ciñéndonos fundamentalmente a los principales acontecimientos sociales y políticos en los Seis Condados a partir del Movimiento pro-Derechos Civiles de 1968, recomendamos al lector interesado en tener una visión más amplia de la génesis de la situación actual el libro de J. C. Beckett, The Making of Modern Ireland, 1603-1923 (Londres, Faber, 1981). En la obra de Paul Arthur, Government and Politics of Northern Ireland (Political Realities Series, Longman, Essex, 1980) se incluye asimismo una relación de los antecendentes del conflicto, aunque es más breve, menos especializada y por consiguiente menos fidedigna que la anterior. Lo que sigue presupone un conocimiento mínimo de la historia irlandesa, sobre todo en lo que se refiere al mini-estado situado al nordeste de la isla.

El problema principal que afronta el observador de hoy es el de decidir si a lo que ocurre en Irlanda del Norte habría o no que llamarle guerra. Al considerar esta cuestión, el lector español haría bien en olvidarse, momentáneamente, del problema social, histórica e ideológicamente distinto, de lo que está pasando en el País Vasco. Cualquier intento de mezclar ambas situaciones conduciría muy probablemente al error, o cuando menos a una simplificación indeseable. Sin embargo, el problema, más general, de terrorismo sigue en pie, y acaso sea éste un punto de partida aceptable para nuestro estudio.

En el espacio de cuatro días, tras el éxito de Sinn Fein al alzarse con el $10 \%$ de los votos y cinco escaños, en las elecciones celebradas en octubre de 1982 para la Asamblea del Ulster, con motivo de la introducción de la llamada Autonomia Progresiva ("Rolling Devolution»), el IRA mató en atentados con bombas a cuatro miembros del Regimiento para la Defensa del Ulster (UDR). El dominical The Sunday Times comentaba asi:

Reclinándose en un sillón en su cómodo despacho de Belfast. Gerry Adams, Vice-Presidente de Sinn Fein, escogió con cuidado sus palabras: "Nosotros suponemos que cada cierto tiempo el IRA realizará una acción de envergadura como parte de la lucha armada". reconoció?.

7 The Sunday Times. 31 de octubre. 1982. pág. 17. 
Este párrafo no por corto es menos significativo. En primer lugar, "Belfast", "Sinn Fein" e "IRA" forman una combinación a la que se viene acostumbrando cada vez más el seguidor de la crisis. Hace ya tiempo que la capital de Irlanda del Norte ha desbancado al Dublín de Tone, O'Connell, Parnell, Connolly, Pearse y de Valera como el centro de la actividad revolucionaria. El Sinn Fein de la época de Bobby Sands poco o nada tiene en común con aquella organización que fundara el capitalista liberal Arthur Griffith hacia principios de siglo, y además el lazo estrecho entre este partido político y el IRA provisional es un secreto a voces, de manera que ese cuidadosamente del periodista es un exceso que casi provoca la risa. No obstante, el periodista no es ningún ingenio: si su reconoció es irónico, la frase con que arranca, reclinándose en su sillón... contrasta sardónicamente con los cuerpos salvajemente destrozados de los miembros del UDR a los que alude en el párrafo anterior. Se nos está pidiendo, insidiosamente, el apoyo, o al menos la compasión, por el UDR legal frente al IRA criminal.

Es más, como la sutileza, por lo visto, ya no se lleva siquiera en el Sunday Times, el artículo está encabezado por tres fotografías que ofrecen apoyo visual para aquellos lectores cuyas pocas luces o mucha prisa les impide captar el mensaje del texto. A la izquierda, un primer plano de la hija - católica, a juzgar por el apellido- de una víctima de la violencia; en el centro, una panorámica de las cruces que conmemoran a los muertos del U/ster; a la derecha, una foto del triunfador Gerry Adams. El pie de la foto reza: Las victimas y el vencedor. El título de tan edificante artículo es: LA POLITICA Y LA MAQUINA DE MATAR.

$\mathrm{Si}$ esto es una muestra de periodismo objetivo, podría pensar Gerry Adams, ¿cómo será la propaganda? Aunque al adicto a la prensa popular se le explican las cosas con más contundencia todavía, la verdad es que el telespectador y el lector británicos están condenados a consumir esta clase de información, presentada de esta manera. Para el mundo de fuera, que quizá haya tomado conciencia últimamente de la maleabilidad y la ductilidad del hierro británico durante el conflicto bélico de las Malvinas, esta insensibilidad no debe de constituir una sorpresa. Lamentablemente, persiste la incomprensión general en lo 
tocante al problema de Irlanda: no parece sino que el público se deje llevar mansamente por la campaña estridente orquestada por la clase política a favor de la Criminalización, Normalización y UIsterización de la crisis. Dicha politica, llevada por los gobiernos laboristas y conservadores indistintamente, ha dado como fruto la aprobación. por administraciones sucesivas, de leves para uso exclusivo de Irlanda del Norte. que podrían parecer duras, incluso. comparándolas con las de los paises más autoritarios pertenecientes al bloque occidental, y que se habrian rechazado con indignación - qué duda cabe - si se hubiese tratado de aplicarlas dentro del territorio de la misma Gran Bretaña.

Algunos ejemplos de esta singular jurisprudencia serían: la reclusión sin juicio (a la que fue sometido Gerry Adams por un período de cuatro años); el no-reconocimiento del status de preso político (lo cual, como es sabido, fue la causa más directa de las huelgas de hambre en la cárcel de Maize en 1981, con un saldo de diez muertos entre los presos del IRA); o esta perla legislativa, incluida en la Ley de Poderes Especiales, vigente hasta hace poco:

Cualquier persona que perpetrare un acto, cuyo efecto fuese intencionadamente perjudicial a la preservación de la paz o al mantenimiento del orden en Irlanda del Norte, y que no estuviese contemplado especificamente en el Reglamento, será considerada culpable de un delito contra el Reglamento. (Sec. 2, |iv|).

Dichas leyes se incrementaban o se modificaban, según lo aconsejaban las necesidades de la crisis creciente o de los criterios internacionales, por medios igualmente anómalos o grotescos. Tal era el caso de los famosos Tribunales Diplock, que suprimian el requisito, que es norma en el resto del Reino Unido, de que los juicios importantes se celebren con juez y jurado. Otro caso de barroquismo forense fue el informe presentado en 1971 por la Comisión Compton, encargada de investigar alegaciones de torturas a prisioneros. El comentarista Kevin Kelley entresaca algunas frases de este informe que, por cierto, fue aprobado en su mayoría por el Tribunal Europeo de los Derechos Humanos:

"Nuestras investigaciones no permiten concluir que ninguno de los que se querella, o como grupo o a título personal, haya sido víctima de ma- 
Ios tratos físicos tal como nosotros entendemos esta expresión", afirma la comisión. En su opinión, para que conste que ha habido malos tratos, seria necesario demostrar que el que los inflige disfruta con el castigo que impone. De lo contrario, según Sir E. Compton, no existen "malos tratos" 8 .

En palabras del líder protestante, Andy Tyrie, Comandante Supremo de la Asociación । para la Defensa del Ulster (UDA):

Existen tribunales especiales y legislación especial; entonces, ¿por qué no puede haber presos especiales también? ${ }^{9}$.

$\mathrm{Ni}$ Margaret Thatcher ni su gobierno encontraron una respuesta válida a tan razonable pregunta. La Ley ha de aplicarse irrevocablemente.

Pero ¿la ley de quién? La respuesta, "de Gran Bretaña», no es muy convincente, ya que desde fines del siglo pasado este país está muy incómodo con su herencia irlandesa. Nadie ha querido cargar con el muerto: ni Liberales, ni Tories ni Laboristas; ni primer ministro británico ni Taioseach (Presidente) de Eire; la patata caliente ha pasado de Fianna Fail a Fine Gael, y de Belfast ha vuelto a Westminster. Y si la respuesta fuera "la ley de Irlanda", entonces preguntaríamos: ¿cuál es el sentido político, hoy, de ese nombre? Las fronteras han avanzado o retrocedido en función de la geografía mental de los presidentes sucesivos: Eamon de Valera, los Cosgrave, Lynch, Haughey y Fitzgerald, como si el destino último de la parcela maldita en el nordeste fuese un secreto de estado, además de un estado secreto. En cuanto a la tantas veces nombrada relación especial entre Dublín y Londres, parece estar basada en un acuerdo, no se sabe si tácito, de que los Seis Condados no se pueden tocar.

Es evidente que Eire no va a aliarse con el Reino Unido contra los nacionalistas republicanos en el norte (aunque la extradición a Irlanda del Norte del militante del Ejército Irlandés de Liberación Nacional [INLA], Dominic McGlinchey, en marzo de es-

${ }^{8}$ Citado en Kevin Kelley, The Longest War: Northern Ireland and the IRA, Zed Press, R. U., 1982, pág. 156.

9 Ibíd., pág. 338. 
te año, y en circunstancias de dudosa constitucionalidad ${ }^{10}$, quizá presagie un futuro maquiavélico en este sentido) mientras que los británicos, por su parte, a pesar de haber mandado a esta zona a casi tantos soldados per capita como los americanos destacaron en Vietnam cuando la guerra, se empeñan en repetir que se trata de un problema de terrorismo que siempre está cerca de una solución final. Sin embargo, el paralelismo con el caso de Vietnam no es muy exacto. En la actualidad, no existe ninguna probabilidad de que los obreros católicos y protestantes se unan para hacer frente a un enemigo común, sea éste inglés o irlandés. Tampoco parece inminente la reunificación de una república irlandesa de 32 condados: los tiempos de Parnell, de Pearse y Connolly, y de Collins han pasado, y no han dejado sucesores.

Por otra parte, Ian Paisley, el predicador fundamentalista con ribetes de neo-nazi, tiene menos posibilidades todavía de capitanear un hipotético ejército lealista en otro glorioso asalto desde las murallas de Derry. Pero el Reverendo "Doctor" afirma impertérrito: "No nos rendiremos: no cederemos ni una sola pulgada». Esta táctica maximalista contribuye a polarizar la opinión en Irlanda del Norte y a mantener el enfrentamiento entre los unionistas a ultranza y los radicales de los "ghettos" católicos, la Falls Road de Belfast y el Barrio de Bogside en Derry. En una situación tan forzada, la esquizofrenia sale a flote, como en esta declaración de un unionista: "Queremos seguir siendo británicos, les guste o no a lós puñeteros ingleses".

Tras una década y media de lucha sorda e incesante, los habitantes de las ciudades de Irlanda del Norte están curados de espantos. Se mueven con facilidad pasmosa entre los escombros y los cascos de los edificios destrozados, y se diría que ni ven, de puro conocidos, a los soldados apostados en las esquinas de las calles de los barrios más afectados. En el Bogside, sobre todo, los muros se han convertido en palimpsestos de hormigón y ladrillos en donde se escriben y se reescriben, a menudo con un humor macabro muy del gusto de este pueblo adusto,

10 Véase el artículo de Mary Holland en The New Statesmen. 23 de marzo. 1984. pág. 6. 
algunos pormenores de esta guerra secreta. Como botón de muestra, sirva esta reflexión, digna de la pluma de Tácito, buen conocedor de los pueblos y paisajes de la bruma céltica:

"¿Existe una vida antes de la muerte?".

En este contexto, la insistencia machacona de Londres en la teoría del terrorismo o del gamberrismo a escala masiva resulta, a la par que cínica, necia. Como indicio de una perturbación más profunda, destaca algo más que el despliegue enorme de tropas y los cambios cruciales efectuados en el Código: me refiero al hecho sin precedentes de que el parlamento autónomo de Stormont -en apariencia modelo de la diplomacia y la representación democrática británicas - tuviese que ser suspendido en 1972. Desde entonces le ha sustituido el régimen de autoridad directa ejercida desde Westminster, si exceptuamos el Acuerdo de Sunningdale (1973-74), ensayo breve y malogrado del concepto de poder compartido. Quizá tengamos una idea más nítida de la profundidad de la división existente en el Norte si recordamos que fue el Movimiento para los Derechos Humanos, de inspiración predominantemente católica, junto con la violencia del IRA, el responsable del derrumbamiento de Stormont, mientras que lo que le asestó el golpe de gracia a Sunningdale fue la huelga lealista provocada por Paisley y el Consejo Unionista Unificado del Ulster.

Parece claro, por tanto, que desde el comienzo de los Troubles (la palabra inglesa significa aproximadamente "embrollos, dificultades, problemas": está visto que con la poderosa riqueza del vocabulario del inglés, se define quien quiere; y, si no, despista y vencerás) los gobiernos británicos sucesivos se han visto obligados por uno u otro bando a ir más allá de lo que les convenía. Asimismo, parece de justicia añadir que Londres se siente más incómodo, por razones obvias, cuando el que lo está empujando es el bloque anti-unionista. Aunque le cueste reconocerlo, Gran Bretaña tiene una guerra entre manos, y esto Dublín, que procura estar siempre al sol que más calienta, lo sabe muy bien.

Curiosamente, en este conflicto entre los dos extremos, nadie ha sabido hacerse fuerte en el terreno de en medio, que teóricamente debería resultar el más atractivo. Asumir la posición li- 
beral es tarea harto dificil en Irlanda del Norte, y la mayoría de los que han intentado practicar la política del borde del abismo, hasta los más cautos, han fracasado. En realidad, desde ambos lados se suelen interpretar tales intentos como un sometimiento cobarde al enemigo o, en el mejor de los casos, como debilidad ante la retórica del contrario. En ambas comunidades, la tradición se paga con la vida, y a este respecto, el IRA, la UDA o incluso el UDR legal no tienen nada que envidiar los unos a los otros. A las transgresiones de orden menor se responde mediante una forma de ostracismo muy rebuscado, y las de mayor cuantía (por ejemplo, si una muchacha nacionalista sale con un soldado del ejército de ocupación) reciben el conocido castigo de embadurnamiento con brea y plumas. Al muchacho que se haya salido de las normas, se le destroza la rótula de un único y certero balazo. (Este kneecapping requiere una mente fría, una mano firme, y el conocimiento íntimo de las armas de fuego de un norirlandés. También es de destacar el rito acentuado que caracteriza a estos castigos.)

Aquí, el asesinato sigue siendo, en la frase famosa del gran iconoclasta irlandés George Bernard Shaw, la forma más extrema de la censura. El brazo de esta "justicia" es largo: Lord Mountbatten murió al explotar una bomba colocada en su yate, en aguas republicanas; otra bomba acabó con la vida del diputado Airey Neave cuando éste se disponia a sacar su coche del aparcamiento de la Cámara de los Comunes; Bernadette Devlin/McAliskey tuvo más suerte, reponiéndose de las heridas de bala recibidas en un atentado del que fue víctima en su casa a primera hora de una mañana de invierno. A cierto nivel, uno tiene que elegir entre diluir sus convicciones, o sucumbir ante ellas; pero hasta en el primer caso, al protestante que exprese su repudio del atentado contra Bernadette Devlin es probable que se le acuse de haberse puesto de parte de los asesinos de Mountbatten, y viceversa.

En el frente puramente político, cualquier claudicación produce víctimas improbables y sincretismos curiosos. O'Neill, Chichester-Clark y Faulkner, a quienes nadie en su sano juicio tildaría de liberales, fueron desbancados por el núcleo de sus propios seguidores conocidos como los naranja sin aditivos. 
Por otra parte, la postura pro-Unión asumida en los últimos tiempos por Gerry Fitt -y que le costó el escaño hace dos añosestá a años luz de la imagen del anti-Unionista resuelto y hasta beligerante de hace algo más de una década, cuando el mundo conoció la identidad del diputado británico ensangrentado tras la paliza administrada por la policía en el transcurso de una manifestación pro-Derechos Civiles en la que participaba. También han visto disminuir su influencia John Hume, del Partido SocialDemócrata y Laborista, en el Norte, y Conor Cruise O'Brien, exministro por Fianna Fail en el Sur, y más tarde director del dominical londinense The Observer. En el caso de O'Brien, cabe pensar que su fracaso se debe al abandono de principios y convicciones anteriores, a su adhesión a un punto de vista en el que sobra frialdad intelectual y falta pasión, y sobre todo a su empeño en elevar a decisiva una parte sólo del conflicto:

\footnotetext{
Volvemos inevitablemente al punto que tantos y tantos intentan negar: al hecho patente de que se trata de una lucha entre grupos definidos por la Religión ${ }^{11}$.
}

Es esta obstinación en olvidar el decisivo aspecto colonialista del problema lo que limita la visión de los liberales y paraliza su acción política. En el caso de Irlanda del Norte, es de importancia crucial reconocer que las líneas de demarcación política coinciden con divisiones de privilegio económico, por escasas que éstas puedan ser, y también que fue parte de la estrategia de los que agenciaron la Partición de la isla el reforzar tales líneas. Dicho en otras palabras, en un intento desesperado de salvar lo que podian de la "debâcle» irlandesa y de aplacar la ira de sus poderosos parientes protestantes, las autoridades británicas prefirieron amputar tres condados del territorio para así garantizar una mayoría unionista en el nuevo "Ulster". Además, basta un vistazo rápido a la ubicación desde entonces de las fábricas y lugares de trabajo para darse cuenta de que la frontera la constituye el río Bann: hacia el este, en el corazón del feudo protestante/unionista formado por los condados de Antrim y Down, se ha concentrado mucha industria nueva, mientras que hacie al oeste, donde los nacionalistas tienen más fuerza, se ha

11 C. C. O'Brien, States of Ireland, Londres, Panther, 1974, pág. 286. 
permitido que las cosas sigan más o menos como antes. En la euforia que siguió a la independencia, Eire volvió la espalda a sus compatriotas del Norte, sin duda viendo en este territorio el puente de plata para el enemigo inglés que huía. Aun así, Collins y los demás republicanos que firmaron el Tratado de Partición, aunque conscientes de la reacción probable de los más radicales - a Collins había de costarle la vida, tal como había previsto- siempre confiaban en que Gran Bretaña renunciase con el tiempo a toda reivindicación territorial sobre Irlanda. Todo esto nos hace pensar que se utilizaron las diferencias religiosas como una segunda y poderosa línea de defensa de los privilegios económicos y sociales de los incondicionales de la Unión, y no al revés.

Los liberales, empero, no han sido los únicos perdedores. Basándose en unos cálculos y suposiciones todavia más extraños, el núcleo del antiguo IRA Oficial, tras olvidarse del marxismo doctrinario de Cathal Goulding (la famosa teoria de etapas) y abandonar la lucha armada, pasó a llamarse Sinn Fein-Partido de los Obreros, que se ha reducido en la actualidad a Partido de Ios Obreros a secas, y es, a juzgar por sus acciones, una organización de convicciones unionistas. Esto parece obedecer a una noción estalinista de lograr el socialismo en un solo pais, pero el problema es que en este caso el tal pais ni existe ni aspira a existir. Efectivamente, ellos y los Paisleyistas son extraños compañeros de cama.

En general, los resultados de las elecciones para la Asamblea tienden a confirmar que en el momento actual la crisis ha llegado a otro punto muerto. Cada una de las facciones se ha dividido en un grupo moderado y otro radical, y la capacidad de maniobra es mayor hacia los extremos que en el centro. La violencia continúa, la situación económica es penosa, y el ejército británico es tan inamovible como el gobierno Thatcher que lo mantiene alli. Expresar siquiera la posibilidad de una solución a esta crisis es arriesgado, pero si ha de haberla, tiene que pasar por el reconocimiento de que el papel de Gran Bretaña es el de una potencia colonialista, de que Dublín ha eludido su antiguo compromiso de lograr una Irlanda reunificada, y de que detrás 
de los gritos de odio e intolerancia mutuos que evidencian el enfrentamiento entre Naranja y Verde, acecha el fantasma de la arrogancia económica, de la protección de intereses creados y privilegios centenarios. No debemos condonar la violencia, pero para poder poner fin a esta guerra, debemos empezar por admitir que existe. 